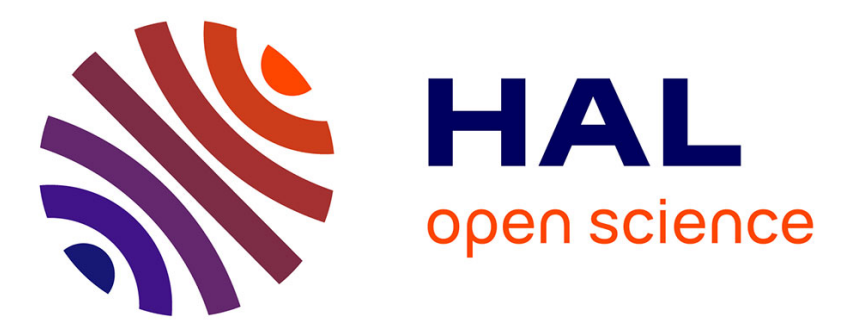

\title{
New Effective Potentials Extraction Method for the Interaction between Cations and Water
}

Xavier Periole, David Allouche, A. Ramírez-Solís, I. Ortega-Blake, J. P. Daudey, Y. H. Sanejouand

\section{> To cite this version:}

Xavier Periole, David Allouche, A. Ramírez-Solís, I. Ortega-Blake, J. P. Daudey, et al.. New Effective Potentials Extraction Method for the Interaction between Cations and Water. Journal of Physical Chemistry B, 1998, 102 (43), pp.8579-8587. 10.1021/jp981688t . hal-02694207

\section{HAL Id: hal-02694207 https://hal.inrae.fr/hal-02694207}

Submitted on 1 Jun 2020

HAL is a multi-disciplinary open access archive for the deposit and dissemination of scientific research documents, whether they are published or not. The documents may come from teaching and research institutions in France or abroad, or from public or private research centers.
L'archive ouverte pluridisciplinaire HAL, est destinée au dépôt et à la diffusion de documents scientifiques de niveau recherche, publiés ou non, émanant des établissements d'enseignement et de recherche français ou étrangers, des laboratoires publics ou privés. 


\title{
New Effective Potentials Extraction Method for the Interaction between Cations and Water
}

\author{
X. Periole, ${ }^{\dagger}, \stackrel{D}{\dagger}$ D. Allouche,$^{\dagger}$ A. Ramírez-Solís,,$^{\ddagger}$ I. Ortega-Blake, ${ }^{\S}$ J. P. Daudey, ${ }^{\dagger}$ and \\ Y. H. Sanejouand $*, \dagger$
}

Laboratoire de Physique Quantique, UMR 5626 of C.N.R.S., I.R.S.A.M.C., Université Paul Sabatier, 118 Route de Narbonne, Toulouse Cedex, 31062, France; Facultad de Ciencias, Universidad Autónoma del Estado de Morelos, Cuernavaca, Morelos, 62290, México; and Laboratorio de Cuernavaca del Instituto de Física, Universidad Nacional Autónoma de México, Apdo. Postal 48-3, Cuernavaca, Morelos, 62251, México

Received: March 31, 1998; In Final Form: July 31, 1998

\begin{abstract}
A very simple method for the extraction of effective interaction potentials from $a b$ initio calculations was proposed (Periole et al. J. Phys. Chem. 1997, 101, 5018), and simple two-body cation-water interaction potentials were derived for several cations, $\mathrm{Li}^{+}, \mathrm{Na}^{+}, \mathrm{K}^{+}, \mathrm{Be}^{2+}, \mathrm{Mg}^{2+}$, and $\mathrm{Ca}^{2+}$, using two facts: first, water molecules in the close vicinity of cations are strongly structured and present a constrained orientation towards the ion; second, at larger distances the ion-water interaction is mainly electrostatic. In the present work, an extension to $\mathrm{Rb}^{+}$and $\mathrm{Sr}^{2+}$ and some refinements of this method are presented. In particular, we explore the most adequate way of including the nonadditivity and polarization effects that arise from the ion-water-water and water-water interactions. The potentials obtained with the new extraction methods are compared with the empirical potentials of Åqvist (Åqvist, J. J. Phys. Chem. 1990, 94, 8021) that were adjusted to reproduce experimental data. Those obtained with the exploration-TIE method are also tested by performing molecular dynamics simulations of the various cation-water systems and the results are found to be in good agreement with experimental data. In particular, they yield cation hydration free energy differences $(\Delta G$ values) that are, in general, in good accordance with experimental figures. This latter method is ideally suited and easy to apply to obtain effective interaction potentials for molecular systems with restricted geometric conditions that appear in numerical simulations, either Monte Carlo or molecular dynamics.
\end{abstract}

\section{Introduction}

The use of numerical simulations for the study of complex molecular systems, e.g. proteins and the chemical behavior of their active sites, is now a common application. One of the limiting factors in these studies is the availability of adequate potentials. On the one hand, they have to be of the simplest possible form, since they will be used in costly simulations in which a large number of atoms is involved and, on the other hand, they have to lead to a reasonable reproduction of the molecular interactions being considered. This has led to the construction of effective two-body potentials, originally for simple systems (for instance, well-known water potentials such as SPC/E, TIP4p, etc.) and now for complex cases where the reduced cost of such potentials can be used advantageously. Recently, a method for easily constructing an effective potential for the interaction of ions with water has been proposed. ${ }^{1}$ The important feature of this method is that the ion remains all the time inside its hydration shell with very particular orientations of the water molecules in its close vicinity. There are a few models taking advantage of the constrained orientation of waters. Cordeiro et al. ${ }^{2}$ proposed a model we shall call "breathing" and is discused below. Bleuzen et al. ${ }^{3}$ proposed a model we shall call single-molecule detachment also discused below, and Sanchez-Marcos and coworkers ${ }^{4-7}$ have developed a model that keeps the hydrated ion either fixed or with a restrained relaxation and construct an interaction of this cluster with water. Recently,

\footnotetext{
* Corresponding author.

† Université Paul Sabatier.

$\doteqdot$ Universidad Autónoma del Estado de Morelos.

$\S$ Universidad Nacional Autónoma de México.
}

Wasserman et al. ${ }^{8}$ took this idea further by considering the hexahydrate as a molecule and describing the interaction energy of the first shell as intramolecular energy, in this way accounting for water relaxation. Floris et al..$^{9-11}$ have developed a method where nonadditivity is accounted for by a polarizable continuum environment where the solute-solvent interaction is computed, producing thus a corrected effective potential. We can say that the idea is quite succesful, leading to a general agreement with experiment even on the solvation energies where earlier works had failed. ${ }^{2,12,13}$ Some of the above models are quite refined and certainly improve the system description. In our previous work, ${ }^{1}$ we used a similar idea by trying to obtain in the most inexpensive manner a very simple potential that can be used for relative comparisons, that is, a simple potential fitted to reproduce the environment and the longer range interactions adequately reproduced by the electrostatic part. In that paper the parameters for the effective potentials describing the interaction of monovalent and divalent cations with an aqueous environment were determined. They were obtained from the results of $a b$ initio calculations of $\mathrm{M}\left(\mathrm{H}_{2} \mathrm{O}\right)_{n}$ systems where $\mathrm{M}$ $=\mathrm{Li}^{+}, \mathrm{Na}^{+}, \mathrm{K}^{+}, \mathrm{Be}^{2+}, \mathrm{Mg}^{2+}$, and $\mathrm{Ca}^{2+}$ and $n=6$, except for $\mathrm{Be}^{2+}$, where $n=4$. These potentials allow us to reproduce the water-cation interaction energy at the Hartree-Fock (HF) level through an analytical form, namely, a sum of two-body LennardJones and electrostatic potentials, the water-water interactions being described using the TIP3p potential. ${ }^{14}$ In that work different forms for the effective potentials were tested and the best fits of $a b$ initio data were obtained with a smooth $r^{-7}$ repulsive and a classical $r^{-4}$ attractive term, in addition to the standard Coulombic interaction. Note that a smooth $r^{-7}$, or $r^{-8}$, 
repulsive term has also been used by other groups, in order to describe accurately alkaline cation-carbonyl oxygen, ${ }^{15}$ lanthanide ions-water oxygen, ${ }^{16}$ as well as $\mathrm{Cr}^{3+}$-water oxygen interactions. $^{7}$ The actual extraction technique used to obtain the corresponding parameters is explained in the Methods section.

Using this effective potential and its associated parameters for molecular dynamics (MD) simulations of $\mathrm{Na}^{+}$and $\mathrm{K}^{+}$in water lead to structural and thermodynamic properties in good accordance with known experimental data. ${ }^{1}$ However, the results obtained from $\mathrm{MD}$ simulations of $\mathrm{Be}^{2+}, \mathrm{Mg}^{2+}, \mathrm{Ca}^{2+}$, and $\mathrm{Li}^{+}$in water were not of the same quality. As a matter of fact, good results were obtained for the cases where the $\mathrm{M}-\mathrm{H}_{2} \mathrm{O}$ $a b$ initio interaction energy is low, namely, about 26 and 18 $\mathrm{kcal} / \mathrm{mol}$ for $\mathrm{Na}^{+}$and $\mathrm{K}^{+}$, respectively. The corresponding interaction energies for $\mathrm{Be}^{2+}, \mathrm{Mg}^{2+}, \mathrm{Ca}^{2+}$, and $\mathrm{Li}^{+}$are about $141,81,55$, and $37 \mathrm{kcal} / \mathrm{mol}$, respectively. Thus, the method proposed previously may work very well only for cations having a low interaction energy with the aqueous environment.

The first goal of the present work was to study the $\mathrm{Sr}^{2+}$ and the $\mathrm{Rb}^{+}$-water systems in order to verify the trends observed previously. Since the description of the cation-water systems first used to obtain the effective potentials proved insufficient to account for large cation-water interactions, we deemed it necessary to take into account not just one but two hydration shells in the model systems from which the effective potential was to be extracted. The basic idea was that such models would reflect in a more realistic way the situation for stronger interacting cation-water systems. We have thus studied $\mathrm{Ca}^{2+}$ and $\mathrm{Mg}^{2+}$ with their first two hydration shells. This, of course, led us to consider new ways to extract the important information from these model systems and to recover the simple two-body effective potential we are looking for. These new methods will be referred to as "exploration" and "breathing" techniques and will be described in the following section. We shall also present there the quantum chemical method used as well as the basis sets and computational details. In the next section we present the results regarding the effective potentials, the MD simulations, and free energy difference calculations that were performed in order to verify their good behavior and the general discussion of these results. We also discuss in that section the problem of whether or not the effective potentials are dependent on the molecular model used to extract them and, if so, to what extent this is important. Various MD calculations are performed using these potentials but with different size of the system in order to check the size dependency of the results. Finally, in the last section, we give our conclusions.

\section{Methods}

1. Quantum Chemical Calculations. For the calculation of molecular interactions between ionic species, the crucial aspect is the correct evaluation of the electrostatic contributions, which are the dominant parts of the interaction. Since most of these contributions are readily included at the HF level, we decided to use this level for the bulk of our calculations. As a matter of fact, in this specific case, the basis set superposition (BSSE) correction (which decreases the interaction energy) is largely compensated by the neglect of post-HF contributions. This is illustrated in ref 1 where we compared our results with more refined calculations in which both BSSE and electronic correlation effects are taken into account. As a matter of fact, in a recent work, Pavlov et al. ${ }^{17}$ have shown that proper description of the dipole moment of water, a crucial characteristic in the ion-water interaction, is attained when the ghost basis set is considered. The overall precision obtained is likely to be sufficient for our purpose which implies a not very detailed analysis of the potential energy surface. On the other hand, maintaining an inexpensive level of theory is in line with the main idea of the present work, which is to test an easy-to-apply method that can be extended to larger systems where refinements such as the inclusion of electronic correlation effects and BSSE corrections are out of the question.

The atomic basis sets come from the TURBOMOLE library, ${ }^{18}$ except for $\mathrm{Ca}^{2+}$, for which an effective core potential of 10 electrons (ecp-10) and the corresponding basis set was derived in previous works. ${ }^{19}$ Since polarization effects are mostly important for the oxygen atom, we chose a TZP (triple- $\zeta+$ polarization) basis including two d functions. We use an effective core potential of 36 electrons (ecp-36) and the corresponding basis for the $\mathrm{Sr}^{2+}$ and the $\mathrm{Rb}^{+} .{ }^{20}$ Thus, basis set sizes are as follows:

$\begin{array}{lll}\mathrm{Sr}^{2+} & (3 \mathrm{~s} 3 \mathrm{p}) /[2 \mathrm{~s} 2 \mathrm{p}] & + \text { ecp-36 } \\ \mathrm{Ca}^{2+} & (6 \mathrm{~s} 8 \mathrm{p} 5 \mathrm{~d}) /[4 \mathrm{~s} 6 \mathrm{p} 2 \mathrm{~d}] & + \text { ecp-10 } \\ \mathrm{Mg}^{2+} & (11 \mathrm{~s} 7 \mathrm{p} 1 \mathrm{~d}) /[6 \mathrm{~s} 3 \mathrm{p} 1 \mathrm{~d}] & \\ \mathrm{Be}^{2+} & (9 \mathrm{~s} 2 \mathrm{p}) /[5 \mathrm{~s} 2 \mathrm{p}] & \\ \mathrm{Rb}^{+} & (3 \mathrm{~s} 3 \mathrm{p}) /[2 \mathrm{~s} 2 \mathrm{p}] & + \text { ecp-36 } \\ \mathrm{K}^{+} & (14 \mathrm{~s} 9 \mathrm{p} 1 \mathrm{~d}) /[9 \mathrm{~s} 5 \mathrm{p} 1 \mathrm{~d}] & \\ \mathrm{Na}^{+} & (11 \mathrm{~s} 7 \mathrm{p} 2 \mathrm{~d}) /[6 \mathrm{~s} 3 \mathrm{p} 2 \mathrm{~d}] & \\ \mathrm{Li}^{+} & (9 \mathrm{~s} 2 \mathrm{p}) /[4 \mathrm{~s} 2 \mathrm{p}] & \\ \mathrm{O} & (9 \mathrm{~s} 5 \mathrm{p} 2 \mathrm{~d}) /[5 \mathrm{~s} 3 \mathrm{p} 2 \mathrm{~d}] & \\ \mathrm{H} & (4 \mathrm{~s} 2 \mathrm{p}) /[2 \mathrm{~s} 2 \mathrm{p}] & \end{array}$

For all the quantum chemical calculations we used the parallel version of GAUSSIAN-94 on the Facultad de Ciencias-UAEM 4-processor SGI-Power Challenge L computer. Quantum chemistry calculations performed previously within the frame of this work were found to be in very good agreement with previous works on these kind of systems, ${ }^{1}$ as are our new calculations on the $\mathrm{Sr}^{2+}-$ and $\mathrm{Rb}^{+}-$water systems. Such calculations are now done following well-established standards, with standard program packages. Moreover, numerous works on cation-water systems have already been performed at the $\mathrm{HF}$ and post-HF levels, allowing for an accurate check of the results obtained.

The form of our effective two-body potential is the following: ${ }^{1}$

$$
\begin{array}{r}
E_{\mathrm{int}}=E_{\mathrm{elec}}+E_{\mathrm{LJ}}=\sum_{i, j}\left\{\frac{q_{i} \cdot q_{j}}{r_{i j}}\right\}+\sum_{(\mathrm{M}, \mathrm{O})}\left\{\frac{A}{r_{\mathrm{MO}}^{7}}-\frac{B}{r_{\mathrm{MO}}^{4}}\right\}+ \\
\sum_{(\mathrm{O}, \mathrm{O})}\left\{\frac{A_{\mathrm{OO}}}{r_{\mathrm{OO}}^{12}}-\frac{B_{\mathrm{OO}}}{r_{\mathrm{OO}}^{6}}\right\}
\end{array}
$$

where $A_{\mathrm{OO}}$ and $B_{\mathrm{OO}}$ are the Lennard-Jones parameters of the TIP3p water model, and where the electrostatic term is calculated with standard charges, namely, $q_{\mathrm{M}}=+1$ or $+2, q_{\mathrm{O}}$ $=-0.834$ and $q_{\mathrm{H}}=+0.417$.

2. Techniques Used To Extract the $\mathrm{M}-\mathrm{H}_{2} \mathrm{O}$ Effective Potential. In this section we shall describe the three techniques used to obtain the $A$ and $B$ parameters of the effective cationwater interaction. The first technique, called single molecule detachment (SMD), was the one used in our previous work, ${ }^{1}$ or in a recent study for extending the original frozen hydrated ion model of Sanchez-Marcos et al. ${ }^{21}$ to produce a fully flexible hydrated ion model for the intermolecular interaction, in order to determine the intracluster interaction potential of $\mathrm{Cr}^{+3}$ $\left(\mathrm{H}_{2} \mathrm{O}\right)_{6} \cdot{ }^{7}$ As mentioned previously, it produced excellent effective $\mathrm{Na}^{+}$-water and $\mathrm{K}^{+}$-water potentials. The molecular system consists of the cation and its first hydration shell with 
4 (in $D_{2 d}$ symmetry for $\mathrm{Be}^{2+}$ and $\mathrm{Li}^{+}$) or 6 (in $T_{h}$ symmetry for all other cations) water molecules. The overall geometry of this $\mathrm{M}\left(\mathrm{H}_{2} \mathrm{O}\right)_{n}(n=4,6)$ system is first optimized at the $\mathrm{HF}$ level. Then a single water molecule is moved along the corresponding $\mathrm{M}-\mathrm{O}$ axis. Thus we obtain the interaction energy of a water molecule and a system composed of the $n-$ 1 remaining water molecules plus the cation, as a function of their distance. We systematically used $12-15$ points to construct a reliable curve. These are actually very few points for a potential surface, but we were also interested in reducing the cost of the parameter extraction to a minimum. Note that, as the $\mathrm{M}-\mathrm{O}$ distance increases, one may go too quickly into the region where the nonadditive effects arising from both subsystems become negligible, henceforth not fully including this information into the effective potential.

The second technique, which we called exploration, allows for obtaining the interaction energy of a cation and one or two hydration shells. This can be represented in a compact notation as $\mathrm{M}-\left(\mathrm{H}_{2} \mathrm{O}\right)_{n}-\left(\mathrm{H}_{2} \mathrm{O}\right)_{m}=(\mathrm{M})_{n, m}$, where $n$ water molecules are in the first hydration shell and $m$ water molecules in the second shell of the cation. In order to obtain the effective interaction energy for different cation-oxygen distances, the position of the cation is changed from its equilibrium geometry obtained at the HF level. These displacements of the cation are the following: \pm 0.2 and $\pm 0.4 \AA$ along the $x, y, z$ axes and along the four $( \pm 1, \pm 1, \pm 1)-(\mp 1, \mp 1, \mp 1)$ lines which are equidistant from the $x, y$, and $z$ axes. This produces 28 different positions lying in two spheres with radii 0.2 and $0.4 \AA$ whose centers are at the equilibrium position of the cation. Of course for symmetric arrangements the potential surface reduces to fewer different values depending on the symmetry. Note that all the water molecules are kept frozen at their optimized geometries. This technique has advantages with respect to the SMD technique, since it can be applied directly to complex biological systems where no reoptimizations can be made as the cation moves inside its first neighbor's sphere. It can also be applied to nonsymmetrical solvation environments such as those coming from MD simulations. As it turns out, it also allows for the introduction, in a better manner than in the previous technique, of the polarization effects on the water molecules due to the presence of the cation as well as of the water-water nonadditive effects. Note that all $\mathrm{M}-\mathrm{O}$ distances are modified at each new position of the cation.

The third technique, called breathing, resembles the first one except that in this case all the water molecules of the first hydratation shell are displaced in a symmetrical manner from their equilibrium positions (as if inflating a balloon). This idea was used by Cordeiro et al. $^{2}$ to produce an effective pair potential. Unfortunately, in their case the interaction between the first hydration shell and the external waters lead to a wrong expansion of the cluster and to an incorrect coordination number. This may be due to the fact that the nonadditivity of the waterwater interaction was not included at the same level of approximation and therefore produced some imbalance in the treatment. Here we found that the technique works fine but care has to be taken with the water-water description. This technique has the added advantage that the high symmetry of the initial model system $\left(T_{h}\right)$ is kept and thus important CPU time savings are possible. Like the SMD technique, it allows for the introduction of the cation-water polarization effects not only as function of the effective cation-water distances but also as a function of water-water distances.

It is possible to define three different types of interaction energy for these cation-water systems. We called the first one "single molecule detachment-interaction energy" (SMD-IE) because of its link to the first technique. This quantity is obtained by subtracting from the total energy of the system (cation plus $n$ water molecules) the energy of an isolated water molecule and the overall energy of the cation plus $(n-1)$ water molecules at their initial equilibrium geometry. This was the definition used in our previous work. It corresponds to the interaction of a semihydrated ion with a single water molecule. For the exploration and breathing techniques we defined two new types of interaction energy: (a) the "total interaction energy" (TIE) which is obtained by subtracting the total energy of the system, the isolated cation energy, and $n$ times the energy of an isolated water molecule in its equilibrium geometry and (b) the "partial interaction energy" (PIE) where we substract from the overall energy the isolated cation energy and the total energy of the water molecules at the geometry of the hydration shell(s) without the cation inside. This latter definition was expected to counterbalance the inaccuracies that could come from the description of water-water interaction energies with the TIP3p potential.

We recall here that $\AA_{\text {quvist }}^{22}$ optimized his parameters for the classical $r^{-12}-r^{-6}$ Lennard-Jones potential in such a way to reproduce, as best as possible, experimentally obtained quantities via molecular dynamics simulations making use of his empirically optimized pair potentials. Note that during these MD simulations he used the SPC potential to account for the waterwater interactions and showed that using the TIP3p model allows to obtain the same results. In fact, the SPC and TIP3p models are very similar. Since we want to compare our effective potential with those obtained by Åqvist, it is natural to use the same TIP3p (or SPC) potential to account for the water-water interactions.

3. Molecular Dynamics and Free Energy Difference Calculations. All the MD simulations were performed at 300 $\mathrm{K}$ with a modified version of the CHARMM-24 program package. ${ }^{23}$ Simulation parameters are standard ones. In particular, bond lengths were constrained with the SHAKE algorithm, ${ }^{24}$ a 2 fs integration time step was used, and nonbonded interactions were calculated with a $14 \AA$ cutoff and a SHIFT truncation procedure for electrostatics. ${ }^{23}$ All LennardJones interactions between the cation and water molecules were taken into account.

The computational model for solvation is as follows: the cation is held fixed at the center of a $15 \AA$ sphere containing 460 TIP3p water molecules. Water molecules lying more than $11 \AA$ away from the cation are also held fixed, as well as water oxygens lying more than $9 \AA$ away. Thus, water molecules in the three first hydration shells of the cation are free to move within a $9 \AA$ radius sphere surrounded by a $2 \AA$ soft boundary, in which water molecules are only free to rotate. This simplified model was designed in order to perform, in a cost-effective manner, free energy perturbation calculations both in water and in a protein environment. Since it is different from the standard fully relaxed model used by Åqvist, it was checked that results obtained with both models are similar. To do so, MD and free energy difference calculations were performed with our water solution model and the parameters and potential energy functions used by Aqqvist in his study, both with the SPC and the TIP3p water models, and we found that the results do agree. ${ }^{1,25}$

Differences of hydration free energies were computed with the thermodynamic perturbation method. The underlying principle of such calculations is as follows: first, several MD simulations are performed during which a cation in a water solution (state "a" of the system) is transformed into another 
TABLE 1: Main Characteristics of the Interaction Energy Curve between a Cation and a Single Water Molecule, As Obtained Using Åqvist's Empirical Potential ${ }^{22}$ or Using the Effective Interaction Potential Obtained with the SMD-IE Method $^{a}$

\begin{tabular}{lllllcc}
\hline & \multicolumn{2}{c}{ Åqvist } & & \multicolumn{3}{c}{ SMD-IE } \\
\cline { 2 - 3 } \cline { 5 - 6 } & $E_{\text {eq }}$ & $R_{\text {eq }}$ & & $E_{\text {eq }}$ & $R_{\text {eq }}$ & msd \\
\hline $\mathrm{Be}^{2+}$ & & & & -97.93 & 1.47 & 1.75 \\
$\mathrm{Mg}^{2+}$ & -70.9 & 1.89 & & -57.40 & 1.92 & 0.70 \\
$\mathrm{Ca}^{2+}$ & -49.7 & 2.28 & & -42.30 & 2.34 & 0.45 \\
$\mathrm{Sr}^{2+}$ & -41.9 & 2.48 & & -38.55 & 2.48 & 0.39 \\
$\mathrm{Li}^{+}$ & -32.6 & 1.95 & & -33.33 & 1.76 & 0.07 \\
$\mathrm{Na}^{+}$ & -23.5 & 2.32 & & -23.46 & 2.23 & 0.07 \\
$\mathrm{~K}^{+}$ & -18.4 & 2.64 & & -16.89 & 2.66 & 0.09 \\
$\mathrm{Rb}^{+}$ & -16.8 & 2.76 & & -17.11 & 2.69 & 0.03
\end{tabular}

${ }^{a}$ Except for $\mathrm{Sr}^{2+}$ and $\mathrm{Rb}^{+}$, the latter results are taken from ref 1 . $E_{\text {eq }}$ is the interaction energy at the equilibrium position, $R_{\text {eq }}$ is the corresponding cation-oxygen distance, and msd is the mean-square difference obtained during our fit of the $a b$ initio data. The energies are in $\mathrm{kcal} / \mathrm{mol}$ and the distances in A. Aqvist did not consider the $\mathrm{Be}^{2+}$ case.

(state "b"), by varying a $\lambda$ parameter in the potential energy function of interaction of the cation with the water molecules. Then, the free energy difference between states $a$ and $b$ is obtained from ${ }^{26}$

$$
\Delta G_{\mathrm{ab}}=\sum_{i} \delta G_{\lambda_{i}}=-k T \sum_{i} \ln \left|\exp \left[-\frac{E\left(\lambda_{i}+\Delta \lambda\right)-E\left(\lambda_{i}\right)}{k T}\right]\right|_{\lambda_{i}}
$$

where $k$ is the Boltzmann constant, $T$ is the absolute temperature, and the brackets indicate that an ensemble average is calculated for each $\lambda_{i}$ value. Note that there is no approximation involved in this equation. From a practical point of view, for each free energy difference calculation, unless stated otherwise, $10 \mathrm{MD}$ simulations at room temperature were performed, each with a given value of $\lambda_{i}$. In each simulation, a 5 ps equilibration period was followed by a $10 \mathrm{ps}$ trajectory, the coordinates obtained at the end of a given simulation being the starting point of the next simulation, performed with a different value of $\lambda_{i}$, namely, $\lambda_{i}+\Delta \lambda$

\section{Results and Discussion}

The most adequate way to compare the parameters obtained for the interaction potentials with the different approaches considered in the present work would have been to test each of them through molecular dynamics and free energy calculations, and to retain those leading to values close to experimental data, for quantities such as radial distribution functions, solvation free energy differences, etc. Since such calculations, and the corresponding analysis, are heavy ones, our choice was to perform them only in the case of a selected set of parameters. To select such a set, in the first part of this work, we use two main quantities that describe, in an approximate manner, the interaction potentials for different cations with a water molecule in $C_{2 v}$ symmetry, namely, the well depth $\left(E_{e q}\right)$, and the cationoxygen equilibrium distance $\left(R_{e q}\right)$, and we compare them to the corresponding values obtained by Åqvist with his empirical approach. $^{22}$ Such a comparison is shown in Table 1 for the parameters obtained in our previous study, ${ }^{1}$ or in the present one, in the case of the $\mathrm{Sr}^{2+}-$ and the $\mathrm{Rb}^{+}-$water systems. Though Åqvist's parameters were determined for a standard 12-6 Lennard-Jones interaction potential, and ours are for a 7-4 one (see the Methods section), the comparison between the corresponding well depth and cation-oxygen equilibrium
TABLE 2: $\quad \mathbf{M}\left(\mathrm{H}_{2} \mathrm{O}\right)_{n, m}$ Systems: Comparison of the Parameters Obtained with the Exploration-PIE and with the Breathing-PIE Method, Applied on ab Initio Geometries with One Hydration Shell, or with Configurations Including One or Two Hydration Shells, Picked from Molecular Dynamics Simulations (MD) ${ }^{a}$

\begin{tabular}{llllll}
\hline \multicolumn{1}{c}{ system } & configuration & method & \multicolumn{1}{c}{$E_{\text {eq }}$} & $R_{\text {eq }}$ & msd \\
\hline $\mathrm{Ca}^{2+}\left(\mathrm{H}_{2} \mathrm{O}\right)_{8,16}$ & MD & Expl-PIE & -42.64 & 2.34 & 2.21 \\
$\mathrm{Ca}^{2+}\left(\mathrm{H}_{2} \mathrm{O}\right)_{8,0}$ & MD & Expl-PIE & -41.32 & 2.35 & 0.39 \\
$\mathrm{Ca}^{2+}\left(\mathrm{H}_{2} \mathrm{O}\right)_{6,0}$ & ab initio & Expl-PIE & -46.01 & 2.33 & 0.0005 \\
$\mathrm{Ca}^{2+}\left(\mathrm{H}_{2} \mathrm{O}\right)_{6,0}$ & ab initio & Br-PIE & -45.85 & 2.35 & 11.49 \\
$\mathrm{Mg}^{2+}\left(\mathrm{H}_{2} \mathrm{O}\right)_{6,18}$ & MD & Expl-PIE & -62.51 & 1.91 & 2.21 \\
$\mathrm{Mg}^{2+}\left(\mathrm{H}_{2} \mathrm{O}\right)_{6,0}$ & MD & Expl-PIE & -61.50 & 1.91 & 1.78 \\
$\mathrm{Mg}^{2+}\left(\mathrm{H}_{2} \mathrm{O}\right)_{6,0}$ & ab initio & Expl-PIE & -64.29 & 1.92 & 0.18 \\
$\mathrm{Mg}^{2+}\left(\mathrm{H}_{2} \mathrm{O}\right)_{6,0}$ & ab initio & Br-PIE & -63.15 & 1.95 & 140.01
\end{tabular}

${ }^{a} n$ is the number of water molecules in the first shell, $m$ is the number of water molecules in the second shell.

distances proved to be useful since the closest values observed in the present work happen to be with parameters leading to calculated quantities closer to experimental data than with the parameter set determined in our previous study (see below).

As shown in Table 1, as far as this criterion is concerned, results close to Åqvist's are obtained for $\mathrm{Rb}^{+}$and $\mathrm{Sr}^{2+}$ cations, which confirm our initial guess, namely, that the SMD-IE method produces good interaction potentials for cases where the cation-water interaction energy is low, i.e., less than 35$40 \mathrm{kcal} / \mathrm{mol}$. In order to explain why this is not the case when the interaction energy becomes larger, two hypotheses were put forward:

1. The poor results obtained for the divalent cations reflect the need for using more realistic models to represent the physical system. In this case it comes down to include the second hydration shell in the model system. The molecules of the second shell should have an influence on those of the first shell and this may allow for reproducing Aqvist's results. In the line of this hypothesis, the agreement with Åqvist's results for the monocations would be due to the fact that for such watercation systems the nonadditive and polarization effects produced

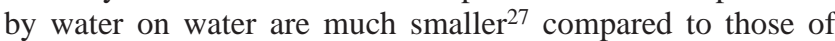
dications.

2. With the SMD technique, it may not be possible to properly reproduce the nonadditivity and polarization effects arising from the water-water and cation-water interactions.

In order to test the first hypothesis, the second hydration shell was included in two models. The geometries of these systems, $\left(\mathrm{Mg}^{2+}\right)_{6,18}$ and $\left(\mathrm{Ca}^{2+}\right)_{8,16}$, were directly picked from molecular dynamics simulations. Since the SMD technique cannot be applied directly in such a case, as a result of the presence of the second water shell, the exploration method was used, and the difference between the parameters obtained with the two methods was assessed by studying the same $a b$ initio geometries as in our previous work, with six water molecules in the first hydration shell of the cations. As shown in Table 2, for both ions, the effect of including the second hydration shell is rather small, leading to increases of 1.3 and $1.0 \mathrm{kcal} / \mathrm{mol}$ of the well depths of $\mathrm{Ca}^{2+}$ and $\mathrm{Mg}^{2+}$, respectively. Hence, the important information that can be obtained with our method, about polarization and nonadditivity effects, is likely to be already included in the first hydration shell. In other words, the effective potentials derived using a single hydration shell seem to be able to reproduce the nonadditivity appearing in the second hydration shell, despite the fact that the latter is certainly important. ${ }^{28}$ In a recent article, Pavlov et al. ${ }^{17}$ have stressed the role of the interaction between the first and second shell of waters. However, in their study they force very symmetric arrangements for both shells which do not happen in the liquid structure 
TABLE 3: $\quad \mathrm{M}\left(\mathrm{H}_{2} \mathrm{O}\right)_{n}$ Systems: Parameters Obtained Using the Exploration-TIE Method Applied on $a b$ Initio Geometries ${ }^{a}$

\begin{tabular}{clllllll}
\hline parameters & \multicolumn{1}{c}{$\begin{array}{c}\mathrm{Sr}^{2+} \\
n=6\end{array}$} & $\begin{array}{c}\mathrm{Ca}^{2+} \\
n=6\end{array}$ & $\begin{array}{l}\mathrm{Mg}^{2+} \\
n=6\end{array}$ & $\begin{array}{c}\mathrm{Be}^{2+} \\
n=4\end{array}$ & $\begin{array}{c}\mathrm{Rb}^{+} \\
n=6\end{array}$ & $\begin{array}{c}\mathrm{K}^{+} \\
n=6\end{array}$ & $\begin{array}{c}\mathrm{Na}^{+} \\
n=6\end{array}$ \\
\hline$A$ & 12195.05 & 8716.88 & 2978.22 & 769.30 & 9379.13 & 10168.35 & 3141.81 \\
$\mathrm{Li}^{+}$ \\
$B$
\end{tabular}

${ }^{a}$ The energies are in $\mathrm{kcal} / \mathrm{mol}$ and the distances in $\AA$.

simulations. As a matter of fact, a recent study ${ }^{29}$ shows that the second shell waters of $\mathrm{Zn}^{2+}$ hydration are the ones responsible for inducing loss of highly symmetrical arrangements.

On the other hand, when the exploration-PIE method is applied on optimized symmetric $a b$ initio geometries or on configurations picked from MD simulations, the results obtained are found to be significantly different (this point will be discussed below). However, the important point is that there is a clear difference between the results obtained with the SMDIE technique, on the one hand, and with the exploration and breathing techniques, on the other hand, the latter ones leading to parameters closer to Åqvist's in both cases considered (see Tables 1 and 2). The fact that the msd with the breathing technique (for $\mathrm{Ca}^{2+}$ as well as for $\mathrm{Mg}^{2+}$ ) are much larger than those obtained with the exploration technique suggests that the details of the large region of the water-water interaction energy surface sampled with the breathing technique is difficult to reproduce with our effective potentials (though it is as well reproduced, on average, as with the exploration technique). Note that most of the configurations considered with the breathing technique are unlikely to occur in a water solution at room temperature.

In the following paragraphs, the exploration method will be studied in depth. Note that this technique is actually the only one, among those we considered, that can be applied in a straightforward way to the case of more complex systems, such as protein binding sites. The advantage here is that with the exploration technique one only needs to know the geometry of the cation environment while, in order to extract the important information for the effective potential, simple moves of the cation along short excursions from its equilibrium position are performed, leaving unchanged the geometry of its environment.

However, the $E_{\mathrm{eq}}$ and $R_{\mathrm{eq}}$ values obtained with the exploration technique for $\mathrm{Ca}^{2+}$ and $\mathrm{Mg}^{2+}$ are still different from those obtained with Åqvist's parameters. This difference, which is larger for the system with the larger interaction energy, may be due to the fact that we attempted to reproduce by an effective cation-water potential the energies of a cluster where the water-water interactions are computed at the $a b$ initio level whereas in the effective potential they are described by the TIP3p water model. As a matter of fact, the eventual waterwater interaction errors due to the TIP3p model (or to the closely related SPC model) were included and accounted for in Åqvist's cation-water potential so as to reproduce experimental data. Thus, it is probably more consistent, within the frame of our approach, to try to describe all the way along the water-water interaction energies with the TIP3p potential. This is the idea underlying the exploration-TIE method (see the Methods section).

In Table $3, E_{\text {eq }}$ and $R_{\text {eq }}$ as well as the parameter values obtained with the exploration-TIE method are shown for all systems studied in our previous work, as well as for the $\mathrm{Sr}^{2+}-$ and the $\mathrm{Rb}^{+}$-water systems. It is obvious that these new results are quite close to those obtained by Åqvist, at least as far as the $E_{\text {eq }}$ and $R_{\text {eq }}$ values are concerned (see Table 1). Indeed, for the seven different cations considered we obtain values for $E_{\text {eq }}$ that are within $2 \mathrm{kcal} / \mathrm{mol}$ of Åqvist's, which is certainly within the limits of the accuracy of our method. As far as the $R_{\text {eq }}$ values are concerned, some small differences can be observed with those obtained by Aqquist, especially in the case of monocations. Note that, in the case of divalent cations, these distances are now in nearly perfect agreement with Åqvist's. Besides the improved correspondence with Åqvist's results, the exploration-TIE method produced smaller msd values than the other methods considered in the present work, in almost all cases studied. For the monovalent cations there is, at least, a 10-fold decrease factor as compared with the original SMD-IE technique. For the divalent cations, the improvement is significant, but impressive only in the $\mathrm{Ca}^{2+}$ case. Note, however, that the range of cation-water distances sampled with the exploration method is much smaller than with the SMD one.

The $\mathrm{Be}^{2+}$ case is a singular one. The analysis of the msd values suggests that the SMD-IE method allows for reproducing the $a b$ initio data in a slightly more accurate way than the exploration-TIE method. This feature may come from the fact that the polarization and nonadditive effects are actually much larger for this system than for the other ones studied. In other words, the TIP3p potential could prove to be too simple when strong effects of this kind are to be taken into account. Such an explanation is supported by a recent $a b$ initio study by Marx et al. where it is suggested that it may not be possible to reproduce the deformations of the water molecules in the first hydration shell of $\mathrm{Be}^{2+}$ with a simple two-body potential. ${ }^{30}$

Our parameters now look close to those obtained by Åqvist, as far as the well depth and the position of the minimum of the interaction between a cation and a single water molecule are concerned. The improvements made in order to develop the exploration-TIE method shed light on two main aspects appearing in the determination of effective potentials. The first one is that special care must be taken in the way the $a b$ initio energy surface is sampled so as to allow for the inclusion of most nonadditive effects in the parameters of the effective potential. Note in particular that various effects can be observed in different physical situations. For instance, the interaction of a cation with a $-\mathrm{COO}^{-}$group within the binding site of a protein is expected to be different, whether the $-\mathrm{COO}^{-}$group is in a mono- or in a bidentate configuration. It is clear that such an effect is expected to be difficult to include in a twobody potential since the oxygen atom polarization is case dependent. The second important point is that during the fitting procedure, the appropriate interaction terms must have been included. In our case, the TIP3p water-water potential used by Åqvist had to be taken into account in order to reproduce his results more accurately.

In order to check the results obtained above, namely, that a model with a second hydration shell is not useful within the frame of the exploration-TIE method, the $\mathrm{Mg}^{2+}\left(\mathrm{H}_{2} \mathrm{O}\right)_{6,0}, \mathrm{Mg}^{2+}$. $\left(\mathrm{H}_{2} \mathrm{O}\right)_{6,18}, \mathrm{Ca}^{2+}\left(\mathrm{H}_{2} \mathrm{O}\right)_{8,0}$, and $\mathrm{Ca}^{2+}\left(\mathrm{H}_{2} \mathrm{O}\right)_{8,16}$ systems were considered again (see Table 4$)$. The $E_{\text {eq }}$ values obtained using one- 
TABLE 4: $\quad \mathrm{M}\left(\mathrm{H}_{2} \mathrm{O}\right)_{n, m}$ Systems: Comparison of the Parameters Obtained Using the Exploration-TIE Method Applied on Systems with One or Two Hydration Shells Picked from MD Simulations

\begin{tabular}{ccccc}
\hline parameters & $\mathrm{Ca}^{2+}\left(\mathrm{H}_{2} \mathrm{O}\right)_{8,0}$ & $\mathrm{Ca}^{2+}\left(\mathrm{H}_{2} \mathrm{O}\right)_{8,16}$ & $\mathrm{Mg}^{2+}\left(\mathrm{H}_{2} \mathrm{O}\right)_{6,0}$ & $\mathrm{Mg}^{2+}\left(\mathrm{H}_{2} \mathrm{O}\right)_{6,18}$ \\
\hline msd & 0.393 & 2.233 & 2.062 & 2.315 \\
$E_{\text {eq }}$ & -45.69 & -41.77 & -67.39 & -59.89 \\
$R_{\text {eq }}$ & 2.31 & 2.35 & 1.87 & 1.92
\end{tabular}

TABLE 5: $\quad \mathrm{M}\left(\mathrm{H}_{2} \mathrm{O}\right)_{n}$ Systems: Comparison of the Parameters Obtained Using the Exploration-TIE Method Applied on $a b$ Initio Optimized Geometries of Systems with Different Numbers of Water Molecules in the First Hydration Shell of the Cation

\begin{tabular}{ccccccc}
\hline & & $n=1$ & $n=4$ & $n=6$ & $n=8$ & $n=10$ \\
cation & & $C_{2 v}$ & $D_{2 d}$ & $T_{h}$ & $D_{4 d}$ & $D_{5 d}$ \\
\hline $\mathrm{Mg}^{2+}$ & $\mathrm{msd}$ & 1.450 & 0.037 & 0.250 & 0.311 & 0.262 \\
& $E_{\text {eq }}$ & -82.92 & -73.77 & -69.23 & -65.76 & -78.77 \\
& $R_{\text {eq }}$ & 1.92 & 1.87 & 1.89 & 1.89 & 1.67 \\
$\mathrm{Ca}^{2+}$ & $\mathrm{msd}$ & 0.502 & 0.006 & 0.0006 & 0.050 & 0.0924 \\
& $E_{\text {eq }}$ & -55.48 & -50.62 & -48.38 & -45.78 & -45.70 \\
& $R_{\text {eq }}$ & 2.26 & 2.28 & 2.31 & 2.32 & 2.26 \\
$\mathrm{Sr}^{2+}$ & $\mathrm{msd}$ & & 0.079 & 0.260 & 0.014 & 0.023 \\
& $E_{\text {eq }}$ & & -41.65 & -40.80 & -39.09 & -38.18 \\
& $R_{\text {eq }}$ & & 2.48 & 2.49 & 2.51 & 2.48 \\
$\mathrm{Li}^{+}$ & $\mathrm{msd}$ & 0.27 & 0.020 & 0.0013 & 0.0905 & \\
& $E_{\text {eq }}$ & -36.93 & -34.80 & -34.39 & -36.83 & \\
& $R_{\text {eq }}$ & 1.80 & 1.81 & 1.81 & 1.70 &
\end{tabular}

and two-hydration shell models are found to be significantly different, both being different from those obtained with ab initio geometries, as previously found while using the explorationPIE method (see Table 2). Thus, here, a paradoxical conclusion is reached: in order to extract effective interaction potentials, the more realistic systems, that is, nonsymmetric systems made of a cation embedded in two hydration shells, are not the best ones. It is possible that the way the studied MD configurations were chosen may explain these unexpected results. Indeed, they were picked from a MD simulation performed with Åqvist's parameters and potential energy function. As a consequence, they are not very low energy ones: the total interaction energies are -308 and $-250 \mathrm{kcal} / \mathrm{mol}$, respectively, for the $\mathrm{Mg}^{2+}\left(\mathrm{H}_{2} \mathrm{O}\right)_{6}$ and $\mathrm{Ca}^{2+}\left(\mathrm{H}_{2} \mathrm{O}\right)_{8}$ systems coming from MD simulations, while they are -326 and $-305 \mathrm{kcal} / \mathrm{mol}$ for these systems when they are optimized in $T_{h}$ and $D_{4 d}$ symmetry. Thus, our method may prove to be efficient only when the configuration of the studied system is a representative one of those sampled at room temperature. This will be checked in future works.

In order to further test how the effective potential depends on the model system, we considered clusters of different sizes, the exploration-TIE technique being applied to the following systems: $\mathrm{Mg}^{2+}\left(\mathrm{H}_{2} \mathrm{O}\right)_{n}, \mathrm{Ca}^{2+}\left(\mathrm{H}_{2} \mathrm{O}\right)_{n}, \mathrm{Sr}^{2+}\left(\mathrm{H}_{2} \mathrm{O}\right)_{n}$ and $\mathrm{Li}^{+}\left(\mathrm{H}_{2} \mathrm{O}\right)_{n}$, $n$ being in the 1-10 range. As shown in Table 5, for a given cation, the $E_{\text {eq }}$ value evolves as a function of the number of water molecules used in the model. Such a trend was also observed in ref 1 with the SMD-IE technique. Here also, these values decrease as $n$ increases and they approach the value obtained by Aqvist when $n=6$. This points again to the fact that a minimum number of water molecules have to be included in the model so that the essential polarization and nonadditive information can be included in the parameters of the effective potential. Note that effective potentials close to Åqvist's and with low msd can be obtained using as few as four water molecules. Interestingly, $n=4$ or 6 corresponds to a number of water molecules close to the number of molecules surrounding most cations in a water solution. Note also that for $\mathrm{Ca}^{2+}$ and $\mathrm{Sr}^{2+}$ the evolution of the $E_{\text {eq }}$ value as a function of $n$ is
TABLE 6: First Maximum $\left(\boldsymbol{R}_{\mathrm{h}}\right)$ and Coordination Number $\left(N_{\mathrm{h}}\right)$ Obtained from the Radial Distribution Functions of Water Oxygens around Cations As Found in Molecular Dynamics Simulations Performed with the 7-4 Lennard-Jones Parameters of Table 3. Comparison with Experimental Data ${ }^{36}$

\begin{tabular}{lclll}
\hline cation & $R_{\mathrm{h}}^{\mathrm{md}}(\AA)$ & $N_{\mathrm{h}}^{\mathrm{md}}$ & $R_{\mathrm{h}}^{\exp }(\AA)$ & $N_{\mathrm{h}}^{\exp }$ \\
\hline $\mathrm{Li}^{+}$ & 1.90 & 4 & $1.94-2.28$ & $4-6$ \\
$\mathrm{Na}^{+}$ & 2.32 & $5-6$ & $2.40-2.50$ & $4-8$ \\
$\mathrm{~K}^{+}$ & 2.83 & $7-8$ & $2.60-2.95$ & $6-8$ \\
$\mathrm{Rb}^{+}$ & 2.86 & $7-8$ & & \\
$\mathrm{Be}^{2+}$ & 1.58 & 4 & 1.67 & 4 \\
$\mathrm{Mg}^{2+}$ & 2.01 & 6 & $2.00-2.15$ & 6 \\
$\mathrm{Ca}^{2+}$ & 2.43 & 8 & $2.33-2.49$ & $6-10$ \\
$\mathrm{Sr}^{2+}$ & 2.58 & 8.5 & $2.60-2.65$ & $8-15$
\end{tabular}

TABLE 7: Protocols Used for $M_{1}$ and $M_{2}$ Free Energy Difference Calculations ${ }^{a}$

\begin{tabular}{ccccc}
\hline protocol & no. of steps & $\Delta \lambda$ & $\begin{array}{c}\text { equilibration } \\
\text { period }(\mathrm{ps})\end{array}$ & $\begin{array}{c}\text { production } \\
\text { period }(\mathrm{ps})\end{array}$ \\
\hline A & 10 & 0.1 & 5 & 10 \\
B & 10 & 0.1 & 10 & 20 \\
C & 10 & 0.1 & 20 & 30 \\
D & 20 & 0.05 & 5 & 10 \\
E & 10 & 0.1 & 5 & 10
\end{tabular}

${ }^{a}$ The A calculation was used for all calculations relative to alkaline cations. The $\mathrm{A}$ and $\mathrm{E}$ calculations only differ in the choice of their initial conditions.

quite smooth (those are known to have a larger number of water molecules in their first hydration shell).

\section{Molecular Dynamics Simulations}

All potentials of Table 3 were tested in molecular dynamics simulations using a modified version of the CHARMM-24 program package in which the 7-4 Lennard-Jones form had been implemented. The $R_{\mathrm{h}}$ and $N_{\mathrm{h}}$ values, corresponding respectively to the first maximum of the cation-oxygen radial distribution function and to the coordination number of the cation, are given in Table 6. They were computed from the last 100 ps of 120 ps simulations. As in our previous work, ${ }^{1}$ they are found to be consistent with experimental data, although calculated $R_{\mathrm{h}}$ values are found to be slightly shorter than experimental ones for the smallest alkaline and alkaline-earth cations (there is up to a 0.09 $\AA$ difference in the case of $\mathrm{Be}^{2+}$ ).

\section{Free Energy Difference Calculations}

As in our previous work, ${ }^{1} \mathrm{Na}^{+}$and $\mathrm{Mg}^{2+}$ were used as starting points of perturbation simulations of alkaline and alkaline-earth cations, respectively. All protocols used are summarized in Table 7 and the main results obtained are given in Table 8.

For each alkaline cation, the protocol used is the one described in the Methods section and the given value is an average between a forward and a backward $\Delta G$ calculation, the accuracy of the calculation being estimated with the hysteresis of the $\mathrm{Na}^{+}$ $\rightarrow \mathrm{Na}^{+}$calculation. For the three transformations studied, it is found to be nearly $0.2 \mathrm{kcal} / \mathrm{mol}$. The results for alkaline cations are all in better agreement with experimental data than those obtained previously, with the set of parameters determined with the SMD-IE method. Notably, both $\mathrm{Li}^{+} \rightarrow \mathrm{Na}^{+}$and $\mathrm{Na}^{+} \rightarrow$ $\mathrm{K}^{+}$cases have been improved. Nevertheless, most of our results are still underestimated with respect to experimental data, especially as far as the $\mathrm{Li}^{+} \rightarrow \mathrm{Na}^{+}$case is concerned. Since $\mathrm{Li}^{+}$is the alkaline cation with the largest interaction energy with water, it is naturally expected to be a difficult case. 
TABLE 8: Differences of Hydration Free Energies of $M_{1}$ and $\mathrm{M}_{2}$ Cations Obtained from Perturbation Simulations Performed with the 7-4 Lennard-Jones Parameters of Table $3^{a}$

\begin{tabular}{lccc}
\hline $\begin{array}{c}\text { transformation } \\
\mathrm{M}_{1} \rightarrow \mathrm{M}_{2}\end{array}$ & $\begin{array}{c}\Delta G_{\mathrm{M}_{1} \rightarrow \mathrm{M}_{2}}^{\text {calc }} \\
(\mathrm{kcal} / \mathrm{mol})\end{array}$ & $\begin{array}{c}\Delta G_{\mathrm{M}_{1} \rightarrow \mathrm{M}_{2}}^{\mathrm{exp}} \\
(\mathrm{kcal} / \mathrm{mol})\end{array}$ & $\begin{array}{c}\Delta G_{\text {old }}^{\text {calc }} \\
(\mathrm{kcal} / \mathrm{mol})\end{array}$ \\
\hline $\mathrm{Li}^{+} \rightarrow \mathrm{Na}^{+}$ & $19.0 \pm 0.2^{b}$ & $26.3-27.5$ & 13.4 \\
$\mathrm{Na}^{+} \rightarrow \mathrm{K}^{+}$ & $15.0 \pm 0.2^{b}$ & $16.7-17.5$ & 20.1 \\
$\mathrm{Na}^{+} \rightarrow \mathrm{Rb}^{+}$ & $21.6 \pm 0.2^{b}$ & 21.5 & \\
$\mathrm{Be}^{2+} \rightarrow \mathrm{Mg}^{2+}$ & $121.7 \pm 5.0^{c}$ & $120.8-135.0$ & 98.5 \\
$\mathrm{Mg}^{2+} \rightarrow \mathrm{Ca}^{2+}$ & $66.3 \pm 1.4^{c}$ & $77.7-80.3$ & 45.7 \\
$\mathrm{Mg}^{2+} \rightarrow \mathrm{Sr}^{2+}$ & $108.4 \pm 0.8^{c}$ & $107.6-113.2$ &
\end{tabular}

${ }^{a}$ Comparison with experimental data ${ }^{37,38}$ and with results obtained with our previous method, $\Delta G_{\text {old }}^{\text {calc } . ~}{ }^{b}$ Hysteresis value. ${ }^{c}$ Rms value on several simulations (see text).

Dication studies required a more detailed analysis. Note that the $\mathrm{Mg}^{2+} \rightarrow \mathrm{Sr}^{2+} \Delta G$ value is in perfect agreement with experimental data, and that both cases studied with our previous method, ${ }^{1}$ have been improved by a large amount (by more than $20 \mathrm{kcal} / \mathrm{mol}$ ). The $\mathrm{Be}^{2+} \rightarrow \mathrm{Mg}^{2+}$ free energy difference obtained with the usual protocol, named A hereafter, is $119.7 \pm 15 \mathrm{kcal} /$ $\mathrm{mol}$; that is, the average calculated value is now very close to the experimental data. However, the hysteresis value for this calculation is quite large. Three other simulations were done in order to improve its accuracy. First, in order to allow the system to equilibrate better, the length of the simulation at each step of the transformation was increased, up to 30 ps $(10+$ $20)$, in the "B" protocol, and to 50 ps $(20+30)$, in the " $\mathrm{C}$ " protocol. The $\Delta G$ values thus obtained are $124 \pm 9$ and 124 $\pm 9.5 \mathrm{kcal} / \mathrm{mol}$, respectively. Second, instead of the 10 steps of protocol A, a 20-step transformation was performed (protocol D), the corresponding $\Delta G$ value being $122 \pm 5 \mathrm{kcal} / \mathrm{mol}$. Though hysteresis values with protocols $\mathrm{B}-\mathrm{D}$ are smaller than with protocol A, they are still not satisfactory. With a different set of initial conditions, another calculation performed with the A protocol yielded a much better hysteresis value: $\Delta G=119.4$ $\pm 0.3 \mathrm{kcal} / \mathrm{mol}$ (protocol "E"). Note that all these results are quite good in spite of the large msd value found during the extraction of the $\mathrm{Be}^{2+}$ interaction potential.

Figure 1, a and b shows, for the "forward" and "backward" calculations, respectively, the $\delta G_{\lambda_{i}}$ value as a function of $\lambda_{i}$ for each of the $\mathrm{A}-\mathrm{E}$ simulations. For the $\mathrm{D}$ one, each value is the sum of $\delta G_{\lambda_{i}}$ and $\delta G_{\lambda_{i}+0.05}$. Along the forward paths, there is a regular increase of $\delta G_{\lambda_{i}}$ up to $\lambda_{i}=0.95$, where a sudden jump is observed. The preliminary increase corresponds to the contraction of the first hydration shell of the cation; that is, average cation-oxygen distances are decreasing, while the free energy jump corresponds to a transition between a state in which there are six water molecules in the first hydration shell of the cation, as in the case of $\mathrm{Mg}^{2+}$, to a state in which there are four such water molecules, as in the case of $\mathrm{Be}^{2+}$ (data not shown). In the A calculation only, the "fifth" and "sixth" water molecules leave the first hydration shell of the cation during the production period of the $\lambda_{i}=0.9$ simulation, while in other calculations they leave it during the corresponding equilibration period (data not shown). However, in all cases, these two water molecules behave in an apparently cooperative manner. In other words, the configurations with five water molecules in the first hydration shell of the cation seem unstable. Along the backward paths, things happen in a quite different way. Indeed, in only one of our backward simulations, namely, the E one, the fifth and sixth water molecules enter the first hydration shell at the same $\lambda_{i}$ value they leave it in the forward simulation. This explains why the hysteresis value was found to be so good in this case $(0.3 \mathrm{kcal} / \mathrm{mol})$ - such a link between larger hysteresis
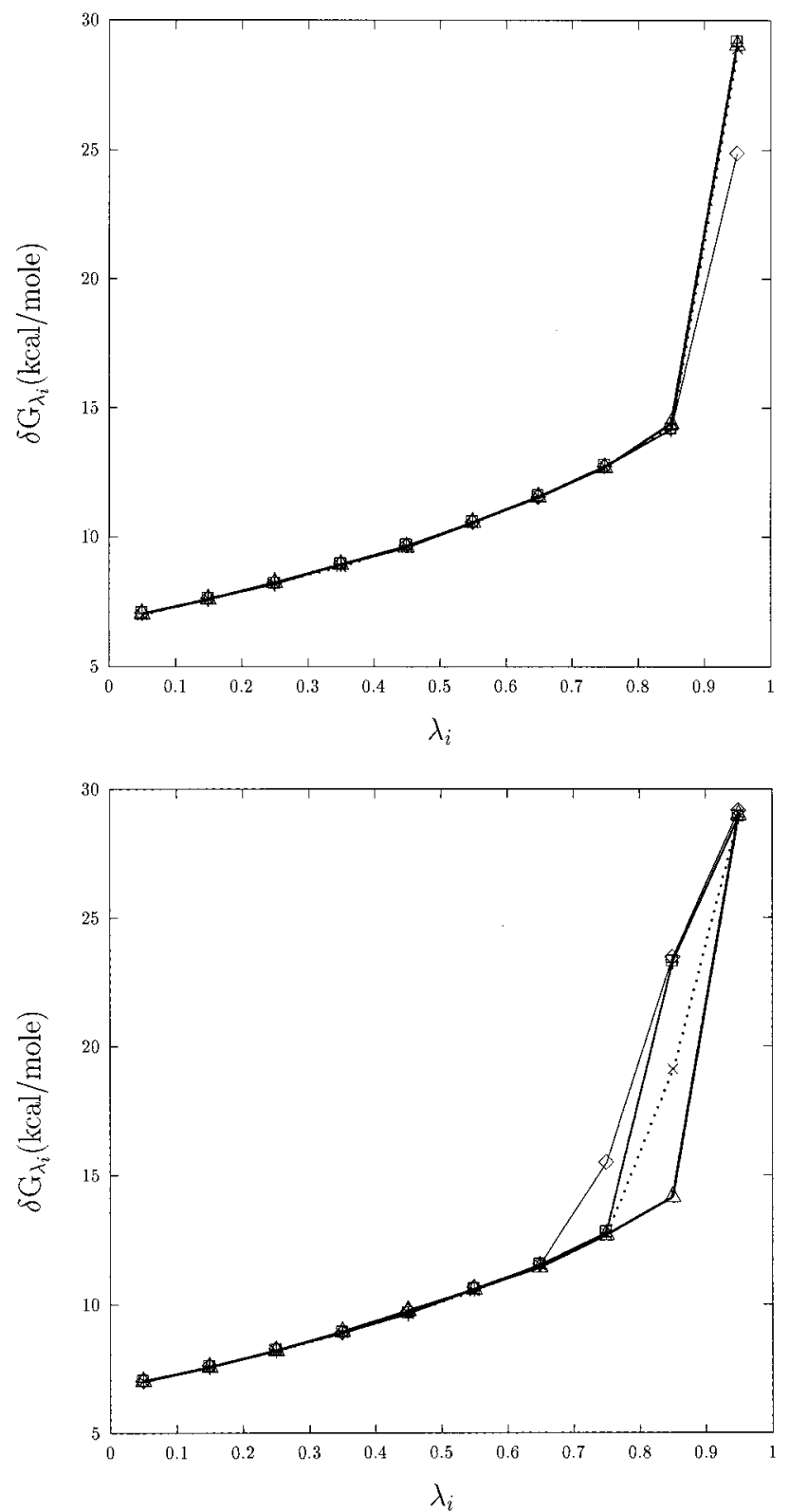

Figure 1. $\delta G_{\lambda_{i}}$ as a function of $\lambda_{i}$ for the $\mathrm{A}-\mathrm{E}$ simulations (see text) of the $\mathrm{Mg}^{2+} \rightarrow \mathrm{Be}^{2+}$ transformation. $\diamond$ corresponds to the A simulation, + to the $\mathrm{B}$ one, $\square$ to the $\mathrm{C}$ one, $\times$ to the $\mathrm{D}$ one, and $\Delta$ to the $\mathrm{E}$ one. (a, top) Forward and (b, bottom) backward calculations.

values and the variation of the water environment in forward and backward calculations at a given $\lambda_{i}$ value was already observed in detailed analysis of $\mathrm{Mg}^{2+} \rightarrow \mathrm{Ca}^{2+}$ transformations. ${ }^{25}$ During three other backward calculations, i.e., the $\mathrm{A}-\mathrm{C}$ ones, both water molecules enter the first hydration shell of the cation at different $\lambda_{i}$ values, ranging from 0.6 to 0.8 . This suggests that it is more difficult to add two water molecules in the first hydration shell of a beryllium-like cation, as in our backward calculations, than to remove them, as in our forward calculations. In other words, in the former case, special initial conditions are likely to be required (for instance, an "expanded" geometry of the four other molecules), that is, the entropic component of the free energy barrier is expected to be large. This will be checked in further works. During the $\mathrm{D}$ backward calculation, the two water molecules enter the first shell at $\lambda_{i}=0.85$; in this case $\Delta \lambda=0.05$ (there are 20 steps in this simulation instead of 10 in the other ones).

To test whether a similar phenomenon is important in the cases of our two other dication transformations, simulations of 
TABLE 9: Differences of Hydration Free Energies of $\mathbf{M g}^{2+}$ and $\mathrm{Ca}^{2+}, \mathrm{Na}^{+}$and $\mathrm{K}^{+}$, Obtained with the Parameters of Table 3, Protocol A, and Systems with Different Numbers of Free Water Molecules around the Cations ${ }^{a}$

\begin{tabular}{crccc}
\hline $\begin{array}{c}\text { transformation } \\
\mathrm{M}_{1} \rightarrow \mathrm{M}_{2}\end{array}$ & $R_{\mathrm{f}}(\AA)$ & $R_{\mathrm{b}}(\AA)$ & $R_{\mathrm{s}}(\AA)$ & $\begin{array}{l}\Delta G_{\mathrm{M} \rightarrow}^{\text {calc }} \mathrm{M}_{2} \\
(\mathrm{kcal} / \mathrm{mol})\end{array}$ \\
\hline $\mathrm{Na}^{+} \rightarrow \mathrm{K}^{+}$ & 9 & 11 & 15 & $15.0 \pm 0.2$ \\
& 11 & 13 & 15 & $15.9 \pm 0.3$ \\
$\mathrm{Mg}^{2+} \rightarrow \mathrm{Ca}^{2+}$ & 12 & 15 & 19 & $13.5 \pm 0.1$ \\
& 9 & 11 & 15 & $66.3 \pm 1.4$ \\
& 11 & 13 & 15 & $67.1 \pm 1.0$ \\
& 12 & 15 & 19 & $65.3 \pm 0.3$
\end{tabular}

${ }^{a} R_{\mathrm{S}}$ is the radius of the system studied, centered on the cation. $R_{\mathrm{f}}$ is the radius of the sphere in which the water molecules are free to move. Water molecules are fixed outside a sphere of radius $R_{\mathrm{b}}$. Otherwise, only their hydrogen atoms are free to move.

TABLE 10: Differences of Hydration Free Energies (kcal/mol) of $\mathrm{Mg}^{2+}$ and $\mathrm{Ca}^{2+}$ Computed with Two Cutoff Values $(3.5 \text { and } 14 \AA)^{a}$

\begin{tabular}{ccc}
\hline & \multicolumn{2}{c}{ parameters origin } \\
\cline { 2 - 3 } cutoff $(\AA)$ & Aqvist & this work \\
\hline 14 & $78.4 \pm 1.6$ & $65.6 \pm 0.1$ \\
3.5 & $79.0 \pm 1.6$ & $79.9 \pm 0.1$
\end{tabular}

${ }^{a}$ Two MD free energy difference calculations were performed, with protocol D, one with the parameters of Table 3 and one with Åqvist's.

$\mathrm{B}$ and $\mathrm{D}$ kinds were performed in both cases. For the $\mathrm{Mg}^{2+} \rightarrow$ $\mathrm{Ca}^{2+}$ transformation, they yield $\Delta G$ values of $65.9 \pm 3.2$ and $65.6 \pm 0.1 \mathrm{kcal} / \mathrm{mol}$, respectively. As in $\mathrm{Mg}^{2+} \rightarrow \mathrm{Be}^{2+} \mathrm{B}$ and $\mathrm{D}$ calculations, the hysteresis of the $\mathrm{D}$ calculation happens to be the smaller one. This suggests that splitting such calculations in a larger number of steps is more efficient than increasing the time span of each step. However, the $\Delta G$ values obtained through $\mathrm{A}, \mathrm{B}$, and $\mathrm{D}$ calculations are all at variance with experimental data by a significant amount (more than $10 \mathrm{kcal} /$ mol). Such a discrepancy is observed in spite of the fact that $\mathrm{Mg}^{2+}-$ or $\mathrm{Ca}^{2+}$ - oxygen potential energy functions used in the present calculations look similar to those proposed by Åqvist, as judged from the location of their minimum (see Tables 1 and 3). In order to understand this point, we first checked that our results do not depend upon the size of the system we considered in our free energy difference calculations, which is different from Åqvist's (see Table 9).

Then, starting from D simulations performed either with our parameters or with Åqvist's, the $\mathrm{Mg}^{2+} \rightarrow \mathrm{Ca}^{2+}$ free energy difference was recomputed, taking only into account water molecules within $3.5 \AA$ of the cation, that is, only those of the first hydration shell. As expected, in this case, there is no significant difference between the results obtained with both kind of parameters (see Table 10), and they are both in good agreement with experimental data. This means that Åvist's parameters were obtained in such a way that the contributions to the free energy difference of water molecules outside the first hydration shell cancel out each other, which is not the case with our potential energy functions. Indeed, our attractive term is a longer range one. Åvist's van der Waals-like interactions are negligible at distances larger than $3.5 \AA$ while ours are not.

On the other hand, the fact that the coordination number of $\mathrm{Ca}^{2+}$ is very dependent on the environment ${ }^{31}$ may explain why it is difficult to reproduce this case. Actually, it was found that in neutron diffraction studies, the number of water molecules in the first hydration shell of $\mathrm{Ca}^{2+}$ is concentrationdependent. ${ }^{32,33}$ Indeed, the deficiencies observed for $\mathrm{Ca}^{2+}$ and $\mathrm{Li}^{+}$can be due to several factors. For instance, the approximations made in the MD simulations (see the Methods section) may have some significant consequences. Also, we have to bear in mind that with the exploration-TIE method the potentials are extracted by adjusting points around the equilibrium distance, whereas in a solution the potential along all $\mathrm{M}-\mathrm{O}$ distances is important. It could be that in these two cases more points of the potential energy surface should be taken into account during the extraction of the parameters. Moreover, the fact that the orientations of the water molecules around the ion are kept highly symmetrical, the cation at its equilibrium position and the atoms of a water molecule being coplanar, may also have some consequences. Obviously for ions where this approximation is less valid, that is, when the first hydration shell waters are more likely to go away from this orientation, ${ }^{32}$ the model may miss some important information. As a matter of fact, even theoretical studies show the lack of rigidity of the molecules in the first hydration shell of $\mathrm{Ca}^{2+}$ as compared to $\mathrm{Mg}^{2+} .34,35 \mathrm{For}^{+} \mathrm{ii}^{+}$the difficulty is even clearer; it is known ${ }^{33}$ that water orientations in its first hydration shell exhibit a strong departure (tilt angle of $\approx 50^{\circ}$ ) from our assumption. All these facts may explain the difficulty of our method to reproduce accurately $\mathrm{Ca}^{2+}$ and $\mathrm{Li}^{+}$properties in solution. In other words, in these cases, the failure of our method may come from not considering the most physically relevant geometrical environment of the cations.

\section{Conclusion}

A new and improved version of an extraction technique proposed previously for determining effective cation-water potentials was developed and tested in molecular dynamics simulations, leading to very good structural and energetic results for many of the studied systems. The improvement of the extraction technique was done in two ways: the introduction of a new approach for the exploration of the potential energy surface from which the effective potential is to be extracted and the definition of a modified interaction energy to be adjusted. It was found that this technique produces effective potentials that converge as the number of water molecules considered in the model system approaches the hydration number.

Molecular dynamics simulations using the presently derived potentials were done to test their ability to describe the hydration of the monovalent and divalent cations. In all cases the first maximum of the cation-oxygen radial distribution function and the coordination number of the cation are found to be in good agreement with experimental data. Free energy difference calculations for the cation-water systems were done using the perturbation method. The results for the previously studied $\mathrm{Li}^{+}$ $\rightarrow \mathrm{Na}^{+}$and $\mathrm{Na}^{+} \rightarrow K^{+}$transformations have been noticeably improved with the new effective potentials. Even if our free energy differences are still underestimated with respect to experimental data, they yield a very good approximation, given the simplicity of the potential used. Large discrepancies were only found for $\mathrm{Li}^{+} \rightarrow \mathrm{Na}^{+}$and $\mathrm{Ca}^{2+} \rightarrow \mathrm{Mg}^{2+}$ cases where, as discussed, the environmental conditions of the $\mathrm{Li}^{+}$and $\mathrm{Ca}^{2+}$ ions in solution were probably not properly accounted for.

We think that one of the most important advantages of this new method of extraction is that it can be easily applied to very complex molecular systems and environments such as those found in the metallic or catalytic sites of proteins where geometric and steric conditions are imposed on the moieties surrounding the cations.

Acknowledgment. We thank Prof. Martin Karplus for kindly providing us with a version of the CHARMM-24 program package, and Prof. Sanchez Marcos and one referee for their 
fruitful suggestions. Grant of computer time c96059, on the IBM-SP2 of the C.N.U.S.C. (Montpellier, France) is acknowledged as well as support via UNAM-DGAPA IN112896 and CONACYT L0044-E9600. A.R. thanks CONACYT for partial support via Project 4061-E for a DEC-Alpha 3000/700 workstation. Partial support from the Ibero-American Program for Science and Technology (CYTED) is also acknowledged via Project V.4 by A.R.

\section{References and Notes}

(1) Periole, X.; Allouche, D.; Daudey, J.-P.; Sanejouand, Y.-H. J. Phys. Chem. 1997, 101, 5018

(2) Cordeiro, M. N. D. S.; Gomes, J. A. N. F. J. Comp. Chem. 1993 14,629

(3) Bleuzen, A.; Foglia, F.; Furet, E.; Helm, L.; Merbach, A. E.; Weber, J. J. Am. Chem. Soc. 1996, 118, 12777.

(4) Sánchez-Marcos, E.; Martínez, J. M.; Pappalardo, R. J. Chem. Phys. 1996, 105, 5968.

(5) Pappalardo, R. R.; Martinez, J. M.; Sanchez Marcos, E. J. Phys. Chem. 1996, 100, 11748 .

(6) Martinez, J. M.; Pappalardo, R. R.; Sanchez Marcos, E. J. Phys. Chem. A 1997, 101, 4444.

(7) Martínez, J. M.; Pappalardo, R. R.; Sánchez-Marcos, E. J. Chem. Phys. 1998, 109, 1445.

(8) Wasserman, E.; Rustad, J. R.; Xantheas, S. S. J. Chem. Phys. 1997, 106,9769 .

(9) Floris, F. M.; Persico, M.; Tani, A.; Tomasi, J. Chem. Phys. Lett. 1992, 199, 518

(10) Floris, F. M.; Persico, M.; Tani, A.; Tomasi, J. Chem. Phys. Lett. 1994, 227,126

(11) Floris, F. M.; Persico, M.; Tani, A.; Tomasi, J. Chem. Phys. 1995 195, 2078.

(12) Clementi, E.; Corongiu, G.; Jonson, B.; Romano, S. J. Chem. Phys. 1980, $72,260$.

(13) Migliore, M.; Corongiu, G.; Clementi, E.; Lie, G. C. J. Chem. Phys. 1988, 88, 7766

(14) Jorgensen, W. L.; Chandrasekhar, J.; Madura, J. D.; Impey, R. W.; Klein, M. L. J. Chem. Phys. 1983, 79, 926.
(15) Roux, B.; Karplus, M. J. Comput. Chem. 1995, 16, 690.

(16) Kowall, T.; Foglia, F.; Helm, L.; Merbach, A. E. J. Am. Chem Soc. 1995, 117, 3790

(17) Pavlov, M.; Siegbahn, P. E. M.; Sandstrom, M. J. Phys. Chem. A 1998, 102, 219.

(18) Ahlrichs, R.; Bar, N.; Haser, M.; Horn, H.; Kolmel, C. Chem. Phys. Lett. 1989, 162, 165.

(19) Ortega-Blake, I.; Barthelat, J. C.; Costes-Puech, E.; Oliveros, E.; Daudey, J. P. J. Chem. Phys. 1982, 76, 4130.

(20) Hay, P. J.; Wadt, W. R. J. Chem. Phys. 1985, 82, 270

(21) Martínez, J. M.; Pappalardo, R.; Sánchez-Marcos, E.; Refson, K.; Diaz-Moreno, S.; Munoz-Paez, A. J. Phys. Chem. B 1998, 102, 3272.

(22) Aqvist, J. J. Phys. Chem. 1990, 94, 8021.

(23) Brooks, B. R.; Bruccoleri, R. E.; Olafson, B. D.; States, D. J.; Swaminathan, S.; Karplus, M. J. Comp. Chem. 1983, 4, 187.

(24) Ryckaert, J. P.; Ciccotti, G.; Berendsen, H. J. C. J. Comput. Phys. 1977, 23, 327.

(25) Allouche, D. Thesis, Univ. Paul Sabatier, Toulouse, France, 1997.

(26) Zwanzig, R. J. Chem. Phys. 1954, 22, 1420.

(27) Clementi, E. Lectures Notes in Chemistry; Springer-Verlag: Berlin, 1980; Vol. 19.

(28) Ortega-Blake, I.; Hernández, J.; Novaro, O. J. Chem. Phys. 1984 81,1894 .

(29) Bernal-Uruchurtu, M. I.; Hernandez-Cobos, J.; Ortega Blake, I., submitted to J. Chem. Phys.

(30) Marx, D.; Sprik, M.; Parrinello, M. Chem. Phys. Lett, 1997, 273 , 360.

(31) Poonia, N.; Bajaj, A. Chem. Rev. 1979, 79, 389.

(32) Hewish, N. A.; Neilson, G. W.; Enderby, J. E. Nature 1982, 297, 138.

(33) Magini, M.; Licheri, G.; Paschina, G.; Piccaluga, G.; Pinna, G. X-ray diffraction on ions in aqueous solutions: Hydration and complex formation; CRC Press: Boca Raton, FL, 1988.

(34) Bernal-Uruchurtu, M. I.; Ortega Blake, I. J. Chem. Phys. 1995 103,1588

(35) Ortega-Blake, I.; Novaro, O.; Les, A.; Rybak, S. J. Chem. Phys. 1982, 76, 5405

(36) Ohtaki, H.; Radnai, T. Chem. Rev. 1993, 93, 1157.

(37) Marcus, Y. Biophys. Chem. 1994, 51, 111.

(38) Gomer, R.; Tryson, G. J. Chem. Phys. 1977, 66, 4413. 